\title{
Modal perturbation method for the dynamic characteristics of Timoshenko beams
}

\author{
Menglin Lou ${ }^{\mathrm{a}}$, Qiuhua Duan ${ }^{\mathrm{a}, \mathrm{b}, *}$ and Genda Chen ${ }^{\mathrm{c}}$ \\ a State Key Laboratory for Disaster Reduction in Civil Engineering, Tongji University, Shanghai 200092, P.R. China \\ ${ }^{\mathrm{b}}$ Civil Engineering Institute, Guangxi University, Nanning 530004, P.R. China \\ ${ }^{\mathrm{c}}$ Department of Civil, Architectural, and Environmental Engineering, University of Missouri-Rolla, Rolla, MO \\ 65409, USA
}

Received 28 December 2004

Revised 5 May 2005

\begin{abstract}
Timoshenko beams have been widely used in structural and mechanical systems. Under dynamic loading, the analytical solution of a Timoshenko beam is often difficult to obtain due to the complexity involved in the equation of motion. In this paper, a modal perturbation method is introduced to approximately determine the dynamic characteristics of a Timoshenko beam. In this approach, the differential equation of motion describing the dynamic behavior of the Timoshenko beam can be transformed into a set of nonlinear algebraic equations. Therefore, the solution process can be simplified significantly for the Timoshenko beam with arbitrary boundaries. Several examples are given to illustrate the application of the proposed method. Numerical results have shown that the modal perturbation method is effective in determining the modal characteristics of Timoshenko beams with high accuracy. The effects of shear distortion and moment of inertia on the natural frequencies of Timoshenko beams are discussed in detail.
\end{abstract}

Keywords: Timoshenko beams, modal perturbation method, modal characteristics

\section{Introduction}

Beams are one of the basic members of large-scale space structures. The lateral vibration of beams is always a concern to civil engineers [1,2]. Depending upon the assumptions introduced in the formulation of the equation of motion, beams can generally be modeled with three theories: Euler-Bernoulli theory, Rayleigh theory, and Timoshenko theory.

In the Euler-Bernoulli beam theory, the rotary inertia and shear deformation of a beam are neglected in the solution of lateral vibration. The theory that takes into account the effect of the rotary inertia was first developed by Rayleigh. Timoshenko extended the Rayleigh theory to include the effects of both rotary inertia and shear deformation. To date, the Timoshenko beam theory is widely applied to describe the flexural vibration of beams $[3,4]$.

The modal perturbation method [5-8], which was developed by expanding any perturbed term as a power series of a small parameter, is an approximate technique for evaluating the effect of a small change in properties of a structural system on its dynamic characteristics and responses. With this method, a set of recurrent formula can be established for the structural system for the determination of its characteristics. The first-order approximation is usually used to derive a numerical solution. Although the second- and higher-order approximations are often tempted to improve the computational accuracy, the third or higher-order analysis in perturbation theory may not be stable and the secondorder approximation does not necessarily render more accurate solutions than the first-order approximation [9]. The

\footnotetext{
${ }^{*}$ Corresponding author. E-mail: lisadqh@ @otmail.com.
} 
direct modal perturbation method that relaxes the assumption for small parameters was developed to improve the stability and accuracy of the conventional perturbation analysis [10]. Numerical simulations have demonstrated that the direct modal perturbation method is very accurate in determining the natural frequencies, mode shapes and modal participation factors of discrete systems. In this paper, the direct modal perturbation method [10] is applied to solve the free vibration problem of a Timoshenko beam. This method utilizes a special Ritz expansion and derivative relation between original and modified systems to simplify the searching process for dynamic characteristics of the modified system. In doing so, the approximate solution of the Timoshenko beam can easily be obtained.

\section{Differential equation for free vibration of Timoshenko beam}

For a uniform Timoshenko beam with a mass per unit length $m$, Young's modulus $E$, and the moment of inertia of the cross section $I$, the undamped free vibration equation can be written as

$$
\begin{gathered}
\partial^{4} y \\
\partial x^{4}
\end{gathered}+\begin{gathered}
m \partial^{2} y \\
E I \partial t^{2}
\end{gathered}-\left(\begin{array}{c}
m r^{2} \\
E I
\end{array}+\begin{array}{c}
m \\
k G A
\end{array}\right) \cdot \begin{gathered}
\partial^{4} y \\
\partial x^{2} \partial t^{2}
\end{gathered}+\begin{gathered}
m^{2} r^{2} \\
k G A E I
\end{gathered} \cdot \begin{aligned}
& \partial^{4} y \\
& \partial t^{4}
\end{aligned}=0
$$

in which $G$ is the shear modulus, and $k$ is the average shear factor depending on the shape of the cross section. Since the beam has uniform properties along its length, the coefficients of Eq. (1) are all constants.

When the beam vibrates in the form of its $i^{\text {th }}$ mode, the transverse displacement $y(x, t)$ can be expressed into $y_{i}(x, t)=\phi_{i}(x) \sin \left(\omega_{i} t+\alpha_{i}\right)$. Substituting it into Eq. (1) gives the following modal governing equation of the uniform Timoshenko beam:

$$
\phi_{i}{ }^{(4)}(x)-{ }_{E I}^{m} \omega_{i}^{2} \phi_{i}(x)+\underset{E I}{m r^{2}}\left(1+\begin{array}{c}
E \\
k G
\end{array}\right) \omega_{i}^{2} \phi_{i}^{(2)}(x)+\underset{k G A E I}{m^{2} r^{2}} \omega_{i}^{4} \phi_{i}(x)=0
$$

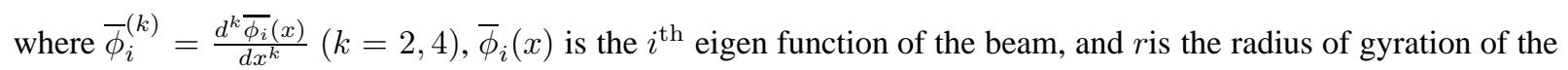
beam cross section. Eq. (2) can be rearranged into:

$$
\phi_{i}^{(4)}(x)+{ }_{E I}^{m r^{2}}\left(1+\begin{array}{c}
E \\
k G
\end{array}\right) \omega_{i}{ }^{2} \phi_{i}^{(2)}(x)+\left(\begin{array}{c}
m^{2} r^{2} \\
k G A E I
\end{array} \omega^{2}-\begin{array}{c}
m \\
E I
\end{array}\right) \omega_{i}{ }^{2} \phi_{i}(x)=0
$$

Let $a=\underset{E I}{m r^{2}}+\underset{k G A}{m}, b=\underset{k G A E I}{m^{2} r^{2}}, c=\underset{E I}{m}$, and $\lambda_{i}=\omega_{i}^{2}$. Equation (3) can be rewritten as

$$
\phi_{i}^{(4)}(x)+a \lambda_{i} \phi_{i}^{(2)}(x)+\lambda_{i}\left(b \lambda_{i}-c\right) \phi_{i}(x)=0
$$

The line over any parameter in Eqs (2)-(4) represents that the parameter is for the Timoshenko beam. When the effects of the rotary inertia and shear deformation are neglected or $a=0$ and $b=0$ in Eq. (4), the modal governing equation of an Euler-Bernoulli beam can be established. That is,

$$
\phi_{i}^{(4)}(x)-c \lambda_{i} \phi_{i}(x)=0
$$

where $\lambda_{i}$ and $\phi_{i}(x)$ are the $i^{\text {th }}$ eigenvalue and eigen function of the Euler-Bernoulli beam, respectively. The solution of Eq. (5) can be expressed into:

$$
\phi_{i}(x)=A \sin p_{i} x+B \cos p_{i} x+C \sinh p_{i} x+D \cosh p_{i} x
$$

in which $p_{i}^{4}=c \lambda_{i}$, the coefficients $A, B, C$ and $D$ can be determined from the boundary conditions of the beam.

\section{Direct modal perturbation method}

To determine the eigenvalues and eigen functions of a uniform Timoshenko beam described by Eq. (4), the direct modal perturbation method is introduced to simplify the process of solution. In this case, the Timoshenko beam is considered as a new or modified system while the Euler-Bernoulli beam that has the same size, shape, and boundary conditions as the Timoshenko beam is an original system. The eigenvalues and eigen functions of the Timoshenko beam can be determined approximately using those of the Euler-Bernoulli beam. Following is a derivation of the 
dynamic characteristics of the Timoshenko beam when the perturbation method is applied to the continuous system. The $i^{t h}$ eigenvalue and eigen function of the Timoshenko beam can be expressed into:

$$
\begin{aligned}
& \phi_{i}(x)=\phi_{i}(x)+\Delta \phi_{i}(x) \\
& \lambda_{i}=\lambda_{i}+\Delta \lambda_{i}
\end{aligned}
$$

The increment $\Delta \phi_{i}(x)$ can be approximately expanded as a sum of the first $n$ lower eigen functions of the original Euler-Bernoulli beam except its $i^{\text {th }}$ eigen function $\phi_{i}(x)$. That is,

$$
\Delta \phi_{i}(x)=\sum_{j=1, j \neq i}^{n} \phi_{j}(x) q_{j}
$$

Equation (9) can be considered as a special Ritz expansion, in which $q_{j}$ is the generalized coordinate for Ritz function $\phi_{i}(x)$. After Eqs (7), (8) and (9) have been introduced, Eq. (4) becomes

$$
\begin{aligned}
& \phi_{i}^{(4)}(x)+\sum_{j=1, j \neq i}^{n} \phi_{j}^{(4)}(x) q_{j}+a\left(\lambda_{i}+\Delta \lambda_{i}\right)\left[\phi_{i}^{(2)}(x)+\sum_{j=1, j \neq i}^{n} \phi_{j}^{(2)}(x) q_{j}\right] \\
& +\left(\lambda_{i}+\Delta \lambda_{i}\right)\left[b\left(\lambda_{i}+\Delta \lambda_{i}\right)-c\right]\left[\phi_{i}(x)+\sum_{j=1, j \neq i}^{n} \phi_{j} q_{j}\right]=0
\end{aligned}
$$

where $\phi_{i}^{(k)}=\frac{d^{k} \phi_{i}(x)}{d x^{k}}$. Introducing Eq. (5) into Eq. (10) results in

$$
\begin{aligned}
& a\left(\lambda_{i}+\Delta \lambda_{i}\right)\left[\phi_{i}^{(2)}(x)+\sum_{j=1, j \neq i}^{n} \phi_{j}^{(2)}(x) q_{j}\right]+b\left(\lambda_{i}^{2}+2 \lambda_{i} \Delta \lambda_{i}+\Delta \lambda_{i}^{2}\right)\left[\phi_{i}(x)+\sum_{j=1, j \neq i}^{n} \phi_{j}(x) q_{j}\right] \\
& -c \Delta \lambda_{i}\left[\phi_{i}(x)+\sum_{j=1, j \neq i}^{n} \phi_{j} q_{j}\right]+\sum_{j=1, j \neq i}^{n} c\left(\lambda_{j}-\lambda_{i}\right) \phi_{j}(x) q_{j}=0
\end{aligned}
$$

By pre-multiplying both sides of Eq. (11) with $\phi_{k}(x)(k=1,2, \cdots, n)$ and integrating the resulting equation over the length of the beam, the $k^{\text {th }}$ equation for the unknown eigenvalue increment $\Delta \lambda_{i}$ and the generalized coordinates $q_{j}(j=1,2, \cdots, n ; j \neq i)$ can be formulated as

$$
\begin{aligned}
& a\left(\lambda_{i}+\Delta \lambda_{i}\right)\left[\int_{0}^{l} \phi_{k}(x) \phi_{i}^{(2)}(x) d x+\sum_{j=1, j \neq i}^{n} q_{j} \int_{0}^{l} \phi_{k}(x) \phi_{j}^{(2)}(x) d x\right]+\left[b\left(\lambda_{i}^{2}+2 \lambda_{i} \Delta \lambda_{i}+\Delta \lambda_{i}^{2}\right)-c \Delta \lambda_{i}\right] \\
& \times\left[\int_{0}^{l} \phi_{k}(x) \phi_{i}(x) d x+\sum_{j=1, j \neq i}^{n} q_{j} \int_{0}^{l} \phi_{k}(x) \phi_{j}(x) d x\right]+\sum_{j=1, j \neq i}^{n} c\left(\lambda_{j}-\lambda_{i}\right) q_{j} \int_{0}^{l} \phi_{k}(x) \phi_{j}(x) d x=0
\end{aligned}
$$

The orthogonal condition of the uniform Euler-Bernoulli beam can be expressed into

$$
\int_{0}^{l} \phi_{k}(x) \phi_{j}(x) d x=e_{k} \delta_{k j}
$$

in which $\delta_{k j}$ is the Knonecker Delta function. After two new parameters, $d_{k j}=\int_{0}^{l} \phi_{k}(x) \phi_{j}^{(2)}(x) d x$ and $e_{k}=$ $\int_{0}^{l} \phi_{k}^{2}(x) d x$, have been introduced, Eq. (12) becomes

$$
\begin{aligned}
& a\left(\lambda_{i}+\Delta \lambda_{i}\right)\left[d_{k i}+\sum_{j=1, j \neq i}^{n} q_{j} d_{k j}\right]+\left[b\left(\lambda_{i}^{2}+2 \lambda_{i} \Delta \lambda_{i}+\Delta \lambda_{i}^{2}\right)-c \Delta \lambda_{i}\right] \\
& \times\left(e_{k} \delta_{k i}+\sum_{j=1, j \neq i}^{n} q_{j} e_{k} \delta_{k j}\right)+\sum_{j=1, j \neq i}^{n} c\left(\lambda_{j}-\lambda_{i}\right) q_{j} e_{k} \delta_{k j}=0
\end{aligned}
$$


Equation (14) can be rearranged into the following non-linear algebraic equation:

$$
\begin{aligned}
& b \Delta \lambda_{i}^{2} \sum_{j=1, j \neq i}^{n} e_{k} \delta_{k j} q_{j}+\Delta \lambda_{i}\left[a \sum_{j=1, j \neq i}^{n} d_{k j} q_{j}+\left(2 b \lambda_{i}-c\right) \sum_{j=1, j \neq i}^{n} e_{k} \delta_{k j} q_{j}+b e_{k} \delta_{k i} \Delta \lambda_{i}\right] \\
& \quad+a \lambda_{i} \sum_{j=1, j \neq i}^{n} d_{k j} q_{j}+b \lambda_{i}^{2} \sum_{j=1, j \neq i}^{n} e_{k} \delta_{k j} q_{j}+\sum_{j=1, j \neq i}^{n} c\left(\lambda_{j}-\lambda_{i}\right) e_{k} \delta_{k j} q_{j} \\
& +\left[a d_{k i}+\left(2 b \lambda_{i}-c\right) e_{k} \delta_{k i}\right] \Delta \lambda_{i}+a \lambda_{i} d_{k i}+b \lambda_{i}^{2} e_{k} \delta_{k i}=0
\end{aligned}
$$

Let $q_{i}=\Delta \lambda_{i} / \lambda_{i}$. Equation (15) can be written in a matrix form when $k=1,2, \cdots, n$

$$
\left(q_{i}^{2}[X]+q_{i}[Y]+[Z]\right)\{q\}+\{w\}=\{0\}
$$

where

$$
\begin{aligned}
& {[X]=\operatorname{diag}\left[b \lambda_{i}^{2} e_{k}\left(1-\delta_{k i}\right)\right]} \\
& {[Y]=\lambda_{i}\left[\begin{array}{cclllll}
a d_{11}+\left(2 b \lambda_{i}-c\right) e_{1} & a d_{12} & \cdots & 0 & \cdots & a d_{1 n} \\
a d_{21} & a d_{22}+\left(2 b \lambda_{i}-c\right) e_{2} & \cdots & 0 & \cdots & a d_{2 n} & \\
\cdots & \cdots & \cdots & \cdots & \cdots & \cdots & \cdots \\
a d_{i 1} & a d_{i 2} & \cdots & b e_{i} \lambda_{i} & \cdots & a d_{i n} & \\
\cdots & \cdots & \cdots & \cdots & \cdots & \cdots \\
a d_{n 1} & a d_{n 2} & \cdots & 0 & \cdots & a d_{n n}+\left(2 b \lambda_{i}-c\right) e_{n}
\end{array}\right]}
\end{aligned}
$$

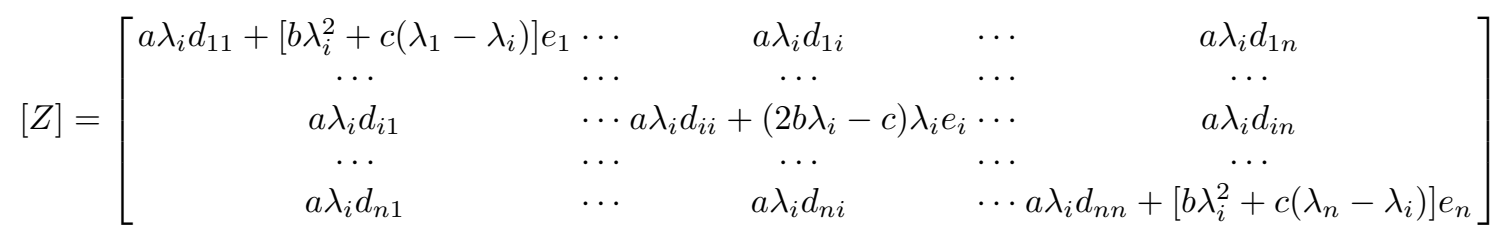

$$
\begin{aligned}
& \{w\}=\left\{\begin{array}{llllll}
a \lambda_{i} d_{1 i} & a \lambda_{i} d_{2 i} & \cdots & a \lambda_{i} d_{i i}+b \lambda_{i}^{2} e_{i} & \cdots & a \lambda_{i} d_{n i}
\end{array}\right\}^{T} \\
& \{q\}=\left\{\begin{array}{llllll}
q_{1} & q_{2} & \cdots & q_{i} & \cdots & q_{n}
\end{array}\right\}^{T}
\end{aligned}
$$

At this point, the partial differential Eq. (4), has been transformed into a set of nonlinear algebraic equations by applying the direct modal perturbation method. It can be clearly seen that Eq. (16) consists of $n$ nonlinear algebraic equations with $n$ unknown variables in the vector $\{q\}$. In general, it is easier to solve the nonlinear algebraic equations than the partial differential equation. The $i^{\text {th }}$ modal frequency and corresponding eigen function can then be obtained by solving the nonlinear algebraic equations iteratively. Obviously, the integrals of $d_{i j}$ and the solution of the nonlinear algebraic equation are main tasks in the modal perturbation method.

To illustrate the application process of the perturbation solution and evaluate its convergence and accuracy, a uniform simply-supported Timoshenko beam of span length $l$ is taken as an example. For a corresponding simplysupported Euler beam, $\lambda_{i}=\omega_{i}^{2}=\underset{m l^{4}}{(i \pi)^{4} E I}, \phi_{k}(x)=\sqrt{ }{ }_{l}^{2} \sin \left(\begin{array}{c}i \pi x \\ l\end{array}\right)$, and then $d_{i j}=-{ }_{l^{2}}^{(i \pi)^{2}} \delta_{i j}$. Thus, Eq. (15) becomes

$$
\begin{aligned}
& b \Delta \lambda_{i}^{2}+\Delta \lambda_{i}\left(a d_{i i}+2 b \lambda_{i}-c\right)+\lambda_{i}\left(a d_{i i}+b \lambda_{i}\right)=0(k=i) \\
& \left(\lambda_{k}-\lambda_{i}\right) q_{k}=0(k=1,2, \cdots, n ; k \neq i)
\end{aligned}
$$

Since $\lambda_{k} \neq \lambda_{i}$ for $k \neq i, q_{k}$ in Eq. (18) must be zero. This means that the shear deformation and rotating inertia have no effect on the flexural vibration mode of the simply-supported Timoshenko beam. However, they affect the natural frequencies of the beam as indicated by a nonzero frequency increment $\Delta \lambda_{i}$ in Eq. (17). The frequency increment can be determined from 


$$
\Delta \lambda_{i}=\frac{1}{2 b}\left[\left(c-a d_{i i}-2 b \lambda_{i}\right)-\sqrt{ }\left(c-a d_{i i}-2 b \lambda_{i}\right)^{2}-4 b \lambda_{i}\left(a d_{i i}+b \lambda_{i}\right)\right]
$$

When the effects of the shear deformation and rotating inertia are taken into account, the natural frequency of the Timoshenko beam is reduced or $\Delta \lambda_{i}$ in Eq. (19) must be negative. Since $\Delta \phi_{i}(x)=0, \phi_{i}(x)$ is equal to $\phi_{i}(x)$. Therefore the solution of $\Delta \lambda_{i}$ determined from Eq. (19) is accurate as $\Delta \lambda_{i}$ is independent of other modes $\phi_{k}(x)$ $(k=1,2, \cdots, \infty, k \neq i)$.

Let us consider the Euler-Bernoulli beam as an extreme case of the corresponding Timoshenko beam when $a=b=0$. In the case, $\Delta \lambda_{i}$ must be zero for $i=1,2, \cdots, \infty$. This observation can be verified from Eq. (19). According to the L, 'Hopital's rule, the limit of the increase in natural frequency when both $a$ and $b$ approach to zero at the same rate, e.g., $a=\gamma b$ ( $\gamma$ is a constant $)$, can be evaluated by

$$
\lim _{a=\gamma b \rightarrow 0} \Delta \lambda_{i}=\lim _{b \rightarrow 0}\left[\begin{array}{c}
-\left(\gamma d_{i i}+2 \lambda_{i}\right)+\left(\gamma d_{i i}+2 \lambda_{i}\right) \\
2
\end{array}\right]=0
$$

It shows that the solution obtained with the direct modal perturbation method is consistent with the analytical solution of the simply-supported Euler-Bernoulli beam. From the above discussions, it is clear that the modal perturbation method results in an exact eigensolution of the simply-supported Timoshenko beam.

\section{Iterative process for solving nonlinear algebraic equations}

For other boundary conditions of the Timoshenko beam, $\bar{\phi}_{i}(x)$ in Eq. (7) generally depends upon all eigen functions $\phi_{k}(x)(k=1,2, \cdots, \infty)$. Therefore, the number of modes must be truncated in order to determine the approximate eigensolution from Eq. (16). After $\Delta \lambda_{i}$ and the generalized coordinates $q_{k}(k=1,2, \cdots, n ; k \neq i)$ in $\{q\}$ have been obtained, the solution of dynamic characteristics for the Timoshenko beam can be solved from Eqs (7) and (8). As Eq. (16) is a nonlinear algebraic equation, an iterative technique must be used to obtain its solution. For this reason, Eq. (16) is rewritten as

$$
f(\mathbf{q})=\left\{f_{1}(\mathbf{q}), f_{2}(\mathbf{q}), \cdots, f_{j}(\mathbf{q}), \cdots, f_{n}(\mathbf{q})\right\}^{T}=\{0\}
$$

where the $j^{\text {th }}$ equation in Eq. (21) becomes

$$
f_{j}(\mathbf{q})=q_{i}^{2} \sum_{k=1}^{n} \lambda_{i}^{2} x_{j k} q_{k}+q_{i} \sum_{k=1}^{n} \lambda_{i} y_{j k} q_{k}+\sum_{k=1}^{n} z_{j k} q_{k}+w_{j}=0
$$

The iterative formula of the Newton method will be used here. It can be described as follows:

$$
\mathbf{q}_{m+1}=\mathbf{q}_{m}-\left(f^{\prime}\left(\mathbf{q}_{m}\right)\right)^{-1} f\left(\mathbf{q}_{m}\right)
$$

in which

$$
\left[f^{\prime}\left(\mathbf{q}_{m}\right)\right]_{j k}=\frac{\partial f_{j}\left(\mathbf{q}_{m}\right)}{\partial q_{k}}
$$

\section{Applications in free vibration characteristics of Timoshenko beams}

Table 1 to Table 3 show the natural frequencies of three Timoshenko beams with different boundary conditions that are commonly seen in engineering practice. The numerical results were obtained by the direct modal perturbation method (MPM) and the finite element method (FEM), respectively, taking into account the effects of the shear deformation and rotating inertia. In the MPM analysis, 20 modes of their corresponding Euler-Bernoulli beam were included. In the FEM analysis, each beam was divided into 10 elements. The FEM computer program used in the numerical examples is Marc Software. The analytical solutions of the corresponding Euler-Bernoulli beam are also listed in the tables for comparison. In all calculations, the Young's modulus $E=2.06 \times 10^{11} \mathrm{~N} / \mathrm{m}^{2}$, the Poisson's ratio $\mu=0.33$, the mass density $\rho=7.85 \mathrm{~kg} / \mathrm{m}^{3}$, the cross section $b \times h=0.3 \mathrm{~m} \times 0.9 \mathrm{~m}$ and the slenderness ratio $\eta=l / r$ is equal to $10,20,30$, and 40 , respectively. 
Table 1

Natural frequencies of the simply-supported Timoshenko beam $(\mathrm{Hz})$

\begin{tabular}{|c|c|c|c|c|c|c|}
\hline Method & $f_{1}$ & $f_{2}$ & $f_{3}$ & $f_{4}$ & $f_{5}$ & $f_{6}$ \\
\hline MPM & 262.2 & 789.9 & 1372 & 1960 & 2542 & 3119 \\
\hline FEM & 265.7 & 810.6 & 1400 & 1984 & 2557 & 3124 \\
\hline Euler-Bernoulli beam & 309.3 & 1237 & 2783 & 4948 & 7732 & 11133 \\
\hline MPM & 73.66 & 262.2 & 512.1 & 789.9 & 1079 & 1372 \\
\hline FEM & 74.09 & 267.0 & 527.9 & 822.5 & 1133 & 1450 \\
\hline Euler-Bernoulli beam & 77.32 & 309.3 & 695.8 & 1237 & 1933 & 2783 \\
\hline MPM & 33.60 & 126.6 & 262.2 & 424.5 & 602.7 & 789.9 \\
\hline FEM & 33.70 & 127.8 & 267.0 & 435.9 & 623.5 & 822.5 \\
\hline Euler-Bernoulli beam & 34.36 & 137.5 & 309.3 & 549.8 & 859.1 & 1237 \\
\hline MPM & 19.08 & 73.66 & 157.2 & 262.2 & 382.1 & 512.1 \\
\hline FEM & 19.12 & 74.09 & 159.0 & 267.0 & 391.5 & 527.9 \\
\hline Euler-Bernoulli beam & 19.33 & 77.32 & 174.0 & 309.3 & 483.2 & 695.8 \\
\hline
\end{tabular}

Table 2

Natural frequencies of the clamped-clamped Timoshenko beam $(\mathrm{Hz})$

$\begin{array}{clcccccc}\eta & \text { Method } & f_{1} & f_{2} & f_{3} & f_{4} & f_{5} & f_{6} \\ 10 & \text { MPM } & 435.0 & 921.6 & 1492 & 2058 & 2717 & 3343 \\ & \text { FEM } & 453.6 & 944.1 & 1515 & 2094 & 2957 & 3745 \\ & \text { Euler-Bernoulli beam } & 701.1 & 1933 & 3788 & 6263 & 9355 & 13066 \\ 20 & \text { MPM } & 148.3 & 354.1 & 601.8 & 873.4 & 1190 & 1500 \\ & \text { FEM } & 150.6 & 359.0 & 613.5 & 892.8 & 1515 & 1829 \\ & \text { Euler-Bernoulli beam } & 175.3 & 483.1 & 947.1 & 1566 & 2339 & 3267 \\ 30 & \text { MPM } & 71.82 & 182.2 & 326.4 & 491.8 & 670.5 & 857.5 \\ & \text { FEM } & 72.37 & 183.8 & 329.9 & 500.0 & 690.0 & 893.6 \\ & \text { Euler-Bernoulli beam } & 77.89 & 214.7 & 420.9 & 695.8 & 1039 & 1452 \\ 40 & \text { MPM } & 41.98 & 110.0 & 203.2 & 315.7 & 443.0 & 582.9 \\ & \text { FEM } & 43.14 & 114.3 & 203.3 & 316.7 & 446.5 & 589.3 \\ & \text { Euler-Bernoulli beam } & 43.82 & 120.8 & 236.8 & 391.4 & 584.7 & 816.6\end{array}$

It is well known that the modal frequencies of a structural system computed with FEM are the upper limit values for the natural frequencies of the system. This statement is verified by the numerical results shown in Tables 1-3. As stated previously, the natural frequencies of the simply-supported Timoshenko beam computed by MPM are exact. Obviously, the calculated values of the natural frequencies of the simply-supported Timoshenko beam obtained from FEM are slightly different from the ones from MPM. Therefore, the proposed modal perturbation method could give results that are more accurate than FEM. At the same time it has been shown from the results in Tables 1 to 3 that the effect of the shear deformation and rotational inertia decreases as the slenderness ratio $(\eta=l / r)$ increases. On the other hand, with the same slenderness ratio, their effect increases as the order of vibration mode becomes higher. More detailed explanations on the effect of the shear deformation and rotation inertia are discussed in the following section.

\section{The effects of shear deformation and rotation inertia}

Let $\alpha_{i}$ be defined as the ratio of the $i^{\text {th }}$ eigenvalue of a Timoshenko beam and an Euler-Bernoulli beam. That is,

$$
\lambda_{i}=\alpha_{i} \lambda_{i}
$$

This coefficient can be used to quantify the effect of the shear deformation and rotation inertia of the Timoshenko beam on its dynamic characteristics. According to Eq. (8), $\alpha_{i}$ can be determined from

$$
\alpha_{i}=1+\begin{gathered}
\Delta \lambda_{i} \\
\lambda_{i}
\end{gathered}=1+q_{i}
$$

Since $\Delta \lambda_{i}$ is negative, the coefficient ranges from zero to one. When it approaches to 1 , the effect is very small and negligible. Otherwise the effect needs to be considered. 
Table 3

Natural frequencies of the cantilever Timoshenko beam $(\mathrm{Hz})$

$\begin{array}{clcccrcc}\eta & \text { Method } & f_{1} & f_{2} & f_{3} & f_{4} & f_{5} & f_{6} \\ 10 & \text { MPM } & 102.0 & 468.6 & 1029 & 1583 & 2091 & 2253 \\ & \text { FEM } & 102.8 & 485.2 & 1070 & 1685 & 2322 & 2960 \\ & \text { Euler-Bernoulli beam } & 110.2 & 690.4 & 1933 & 3789 & 6262 & 9355 \\ 20 & \text { MPM } & 26.68 & 151.8 & 374.2 & 639.9 & 930.6 & 1232 \\ & \text { FEM } & 27.05 & 153.9 & 382.5 & 659.1 & 964.5 & 1286 \\ & \text { Euler-Bernoulli beam } & 27.54 & 172.6 & 483.3 & 947.2 & 1566 & 2339 \\ 30 & \text { MPM } & 12.03 & 72.14 & 188.2 & 338.8 & 513.3 & 704.5 \\ & \text { FEM } & 12.14 & 72.67 & 190.8 & 346.1 & 527.9 & 728.4 \\ & \text { Euler-Bernoulli beam } & 12.24 & 76.71 & 214.8 & 421.0 & 695.8 & 1039 \\ 40 & \text { MPM } & 6.80 & 41.15 & 111.6 & 206.9 & 322.1 & 452.7 \\ & \text { FEM } & 6.85 & 41.83 & 112.7 & 210.1 & 329.0 & 464.9 \\ & \text { Euler-Bernoulli beam } & 6.89 & 43.65 & 120.8 & 236.8 & 391.4 & 584.7\end{array}$

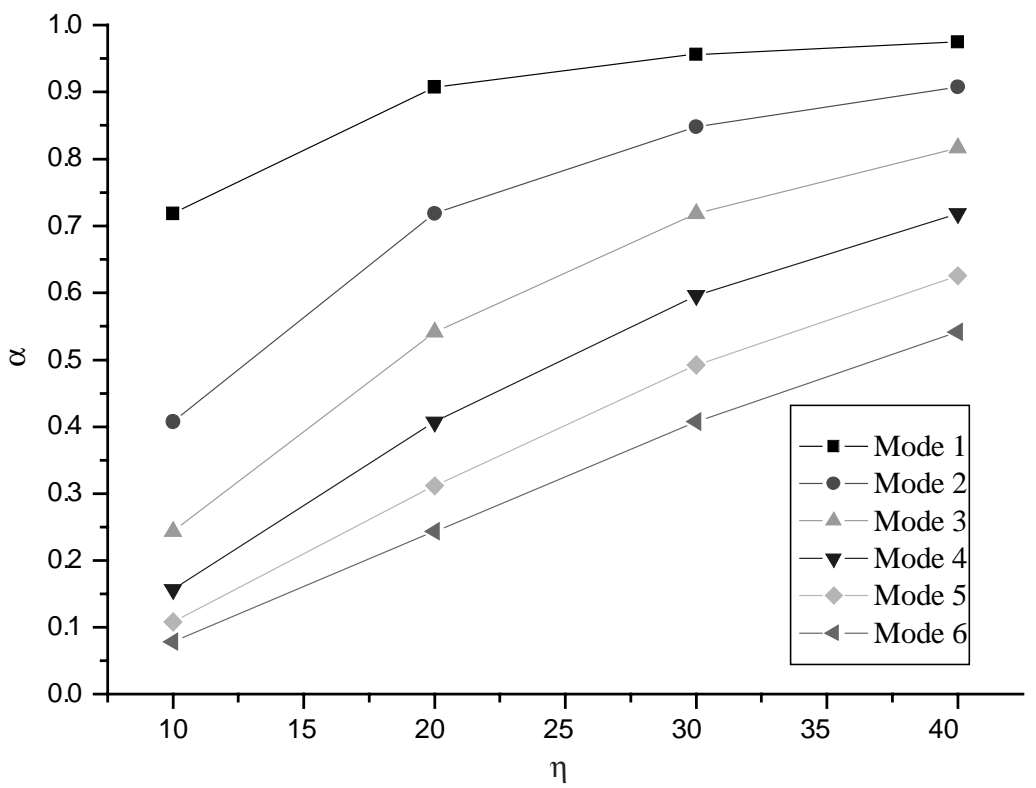

Fig. 1. The relation between $\alpha$ and $\eta$.

The coefficient $\alpha_{i}$, defined in Eq. (24), is for the combined effects of the shear deformation and rotation inertia of a Timoshenko beam, representing the difference between the Timoshenko beam theory and the Euler beam theory. To understand their individual effect, another coefficient, $\beta_{i}$, is defined as the ratio of the $i^{\text {th }}$ frequency reductions due to the shear deformation and rotation inertia effects, respectively. It can be expressed into:

$$
\beta_{i}=\left|\Delta \omega_{i}^{s} / \Delta \omega_{i}^{r}\right|
$$

in which $\Delta \omega_{i}^{s}=\omega_{i}^{s}-\omega_{i}$ and $\Delta \omega_{i}^{r}=\omega_{i}^{r}-\omega_{i}$ represent the frequency reductions by taking into account the effect of shear deformation and the effect of rotation inertia, respectively.

The coefficients $\alpha_{i}$ and $\beta_{i}$ as a function of the slenderness ratio $l / r$ are presented in Figs 1-6 for the simplysupported, clamped-clamped and cantilever Timoshenko beams, respectively. It can be seen from Figs 1, 3 and 5 that the first eigen value of the Timoshenko beam approaches to that of the Euler beam only when the slenderness ratio $l / r \geqslant 30$. For all higher-order modes, the frequencies of the Timoshenko beam are quite different from those of the Euler beam. The higher the order of a mode, the more significant the effects of shear deformation and rotation inertia will be. This means that, in addition to the slenderness ratio, the order of mode is also an important factor to determine whether the effects of shear deformation and rotation inertia can be neglected. Furthermore, the excitation 


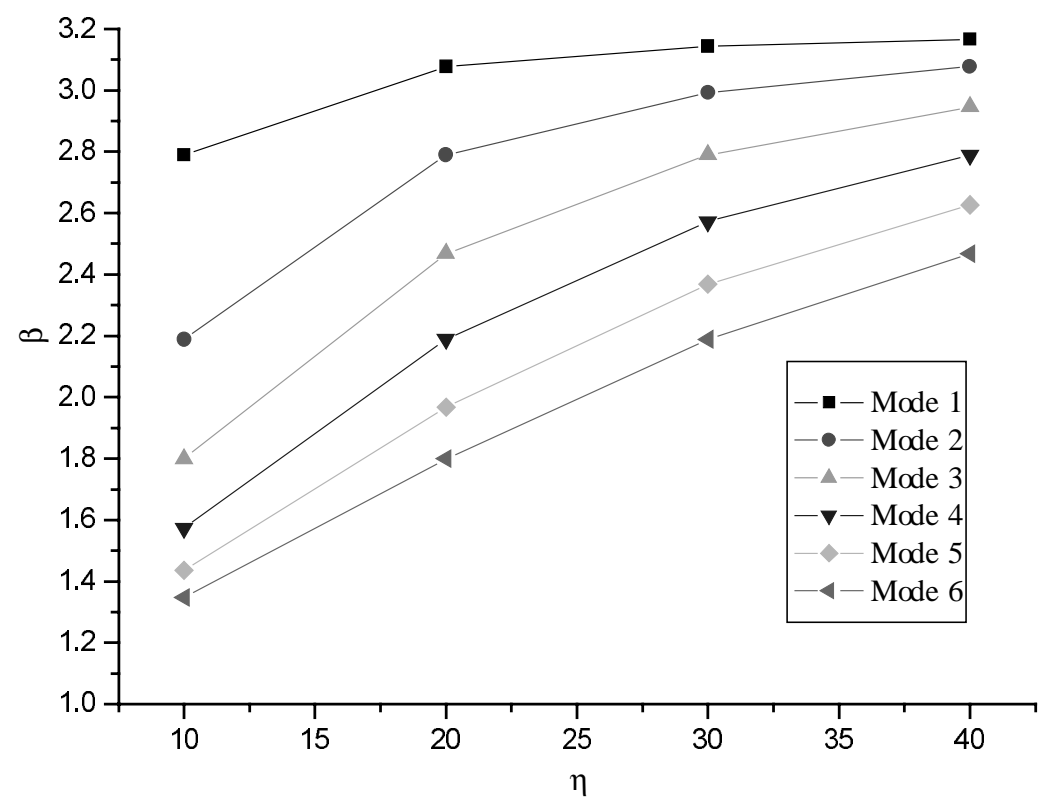

Fig. 2. The relation between $\beta$ and $\eta$.

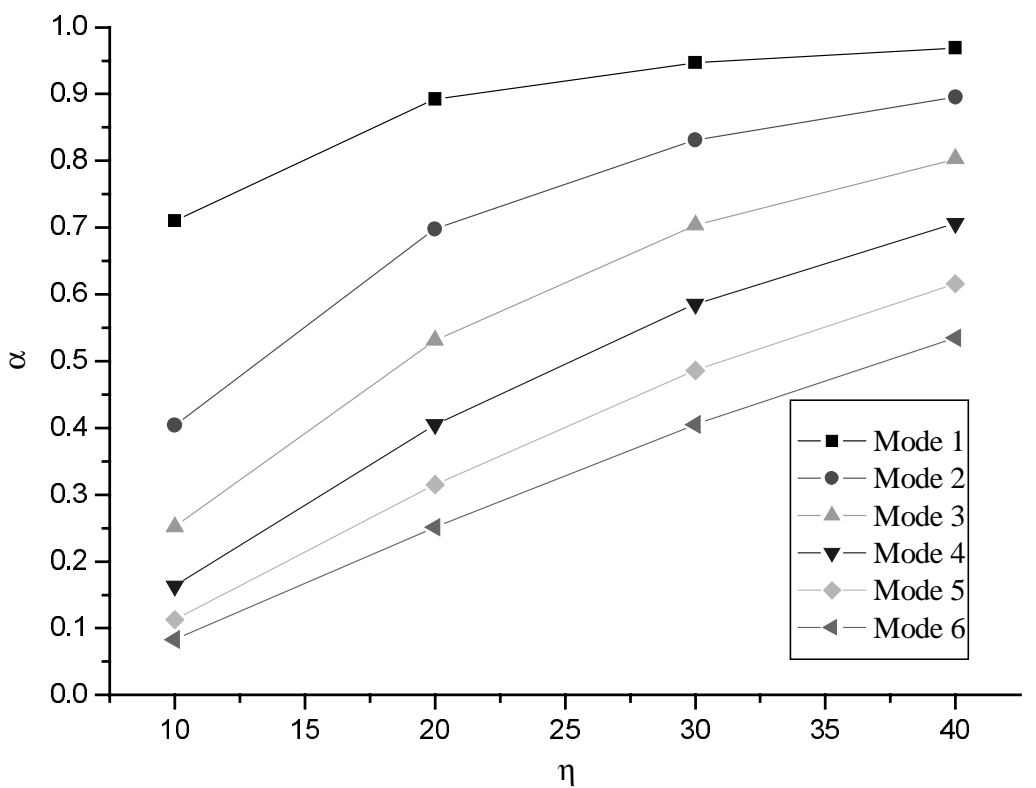

Fig. 3. The relation between $\alpha$ and $\eta$.

frequency of a dynamic load must also be a determining factor in the assessment of shear deformation and rotation inertia effects due to potential resonant effects.

It can be observed from Figs 2, 4 and 6 that the frequency reduction due to the presence of the shear deformation is greater than that of the rotary inertia. This reduction becomes more pronounced as the order of mode becomes higher and the slenderness ratio $l / r$ decreases. 


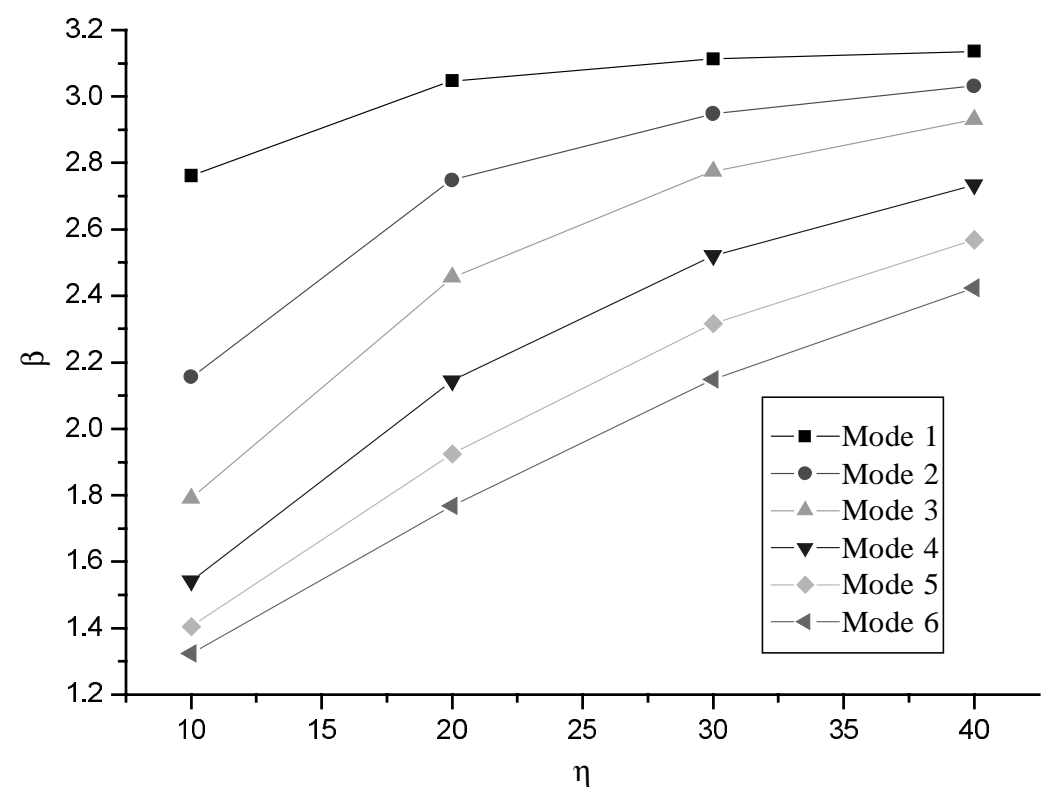

Fig. 4. The relation between $\beta$ and $\eta$.

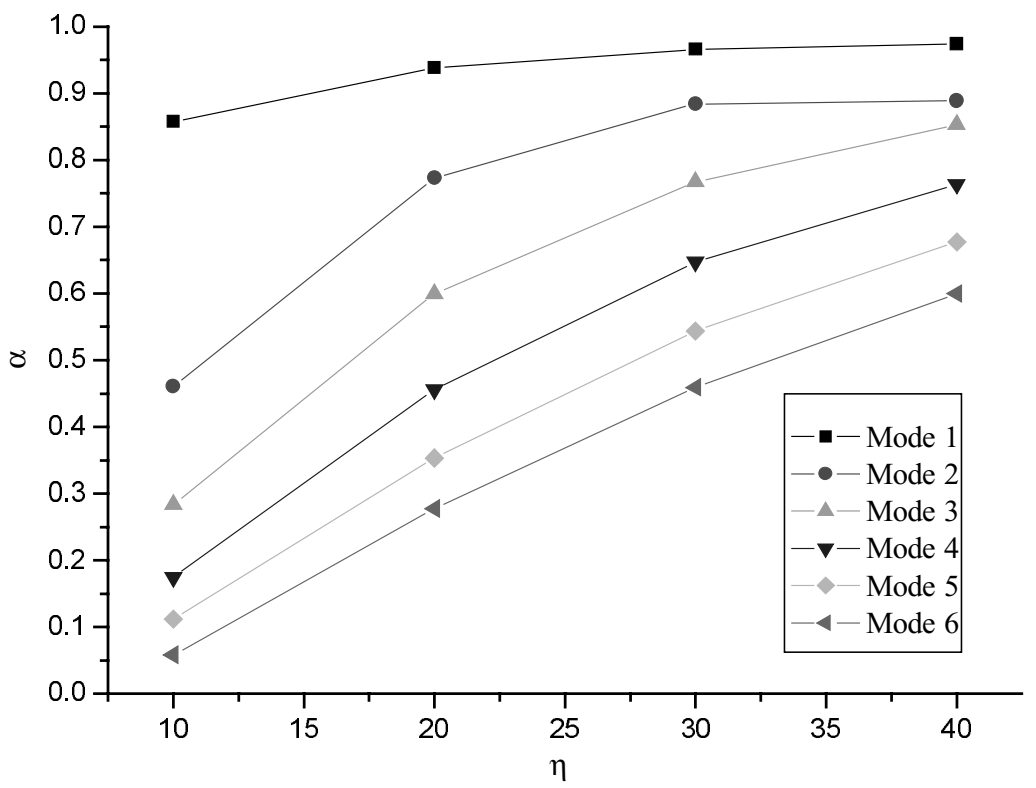

Fig. 5. The relation between $\alpha$ and $\eta$.

\section{Conclusions}

In this paper the direct modal perturbation method based on the Ritz expansion has been applied to solve for the dynamic characteristics of a Timoshenko beam. The eigen functions of its corresponding Euler beam was chosen as Ritz functions. Therefore the undamped free vibration equation of the Timoshenko beam can be converted into a set of nonlinear algebraic equations. Numerical results from several examples have shown that the perturbation method is accurate, computationally efficient, and applicable to Timoshenko beams of any support conditions. The lower-order natural frequencies determined by this method are more accurate than those of the higher modes. The 


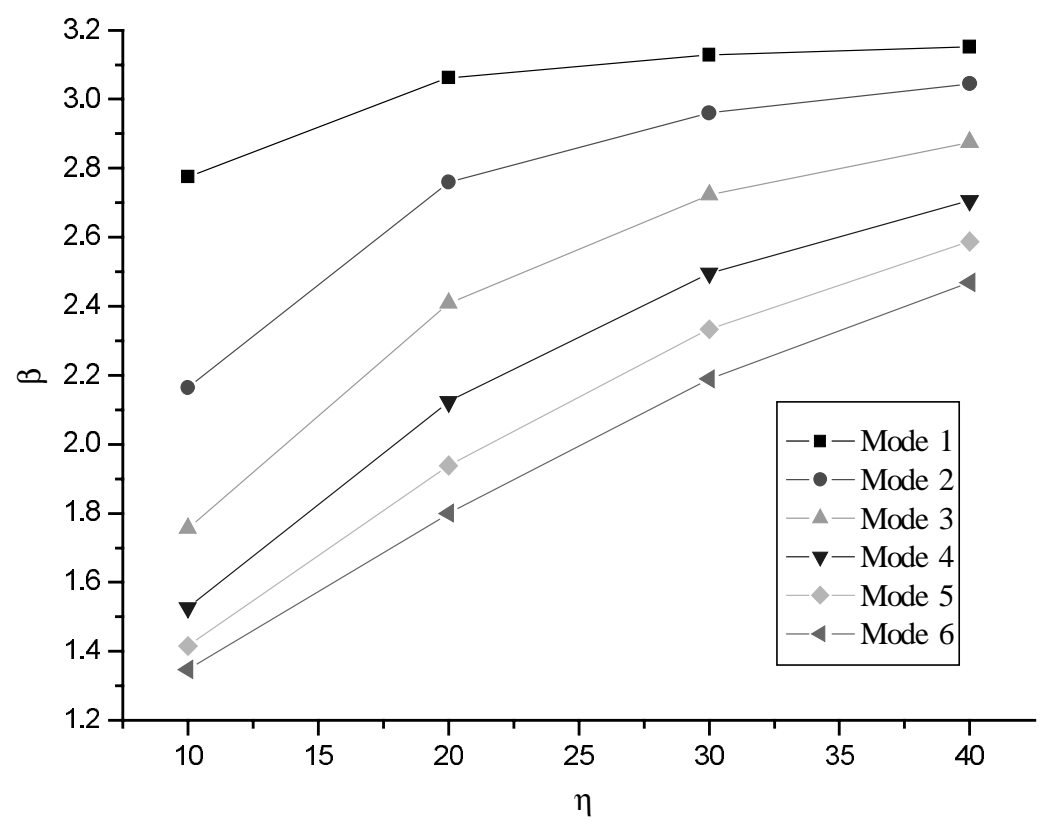

Fig. 6. The relation between $\beta$ and $\eta$.

effects of shear deformation and rotation inertia are negligible when the slenderness ratio of the beam is large. The difference between the natural frequencies of a Timoshenko beam and its corresponding Euler beam increases as the order of mode becomes higher.

\section{Acknowledgment}

This research was sponsored by the National Natural Science Foundation of China through Grant No. 50279031. This support is gratefully acknowledged.

\section{References}

[1] R.W. Clough and J. Penzien, Dynamics of structures, (2th ed.), New York: McGraw-Hill, Inc., 1993.

[2] F.Y. Cheng, Matrix analysis of structural dynamics, New York: Marcel Dekker, Inc., 2000.

[3] S. Timoshenko, D.H. Young and W. Weaver, Jr., Vibration problems in engineering, (4th ed.), New York: John Wiley \& Sons, Inc., 1974.

[4] R.A. Anderson, Flexural vibrations in uniform beams according the Timoshenko theory, Journal of Applied Mechanics 75 (1953), 504-510.

[5] J.H. Wilkinson, The algebraic engineering problem, Oxford: Oxford Clarendon Press, 1965.

[6] L. Meirovitch, Perturbation method, New York: Wiley, 1973.

[7] A.H. Nayfeh, Introduction to perturbation techniques, New York: John Wiley \& Sons, 1981.

[8] Z. Lu, Z. Feng and C. Fang, Matrix perturbation method in modal coordinate system for linearly generalized eigenvalue problem, J. of Vibration Engineering 2(2) (1989), 59-64, in Chinese.

[9] G. Chen and G. Lin, Dynamic interaction between substructures, Journal of Northeast Hydraulic Power and Electricity 4(2) (1988), 1-10, in Chinese.

[10] M. Lou and G. Chen, Modal perturbation method and its applications in structural systems, Journal of Engineering Mechanics ASCE 169(8) (2003), 935-943. 

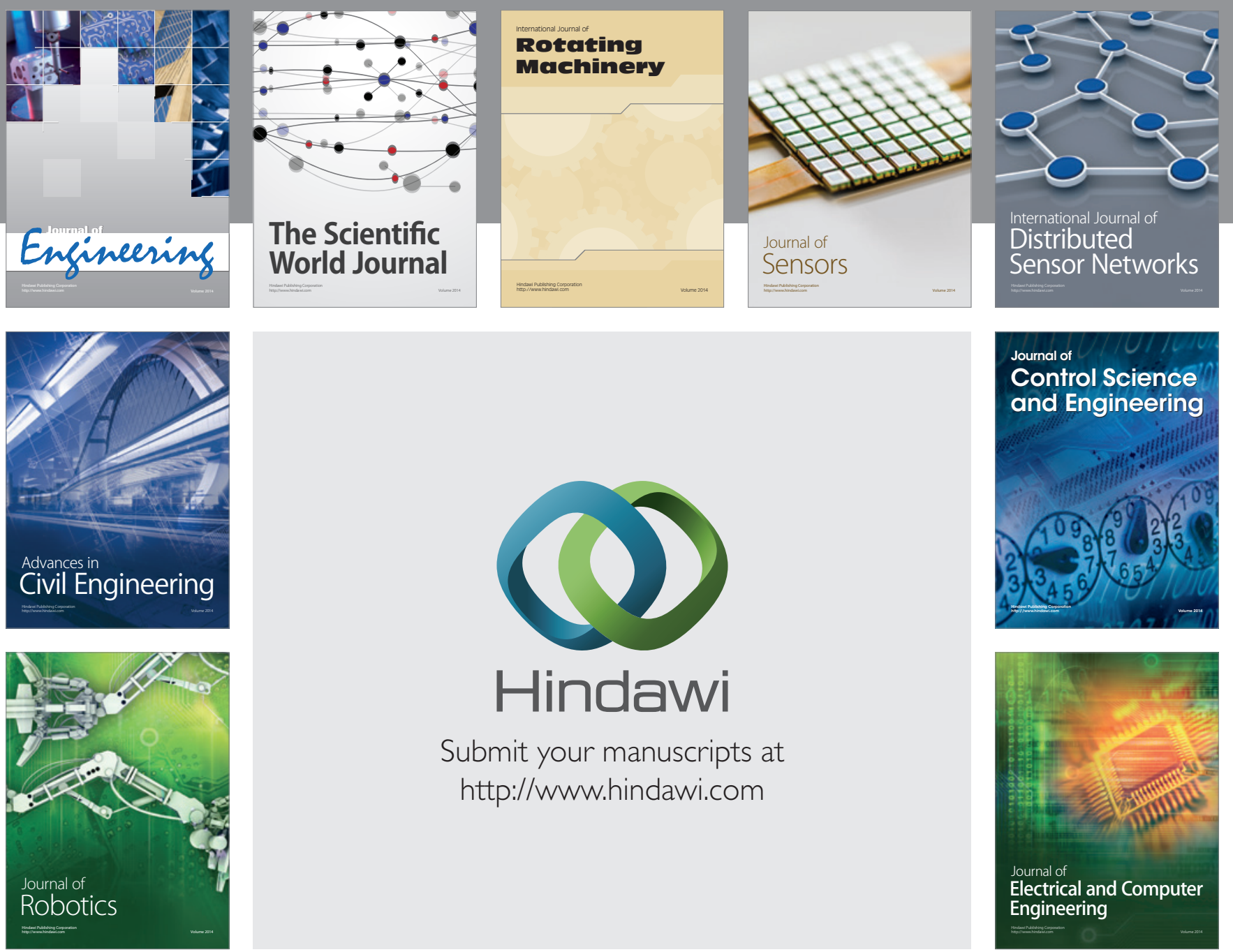

Submit your manuscripts at

http://www.hindawi.com
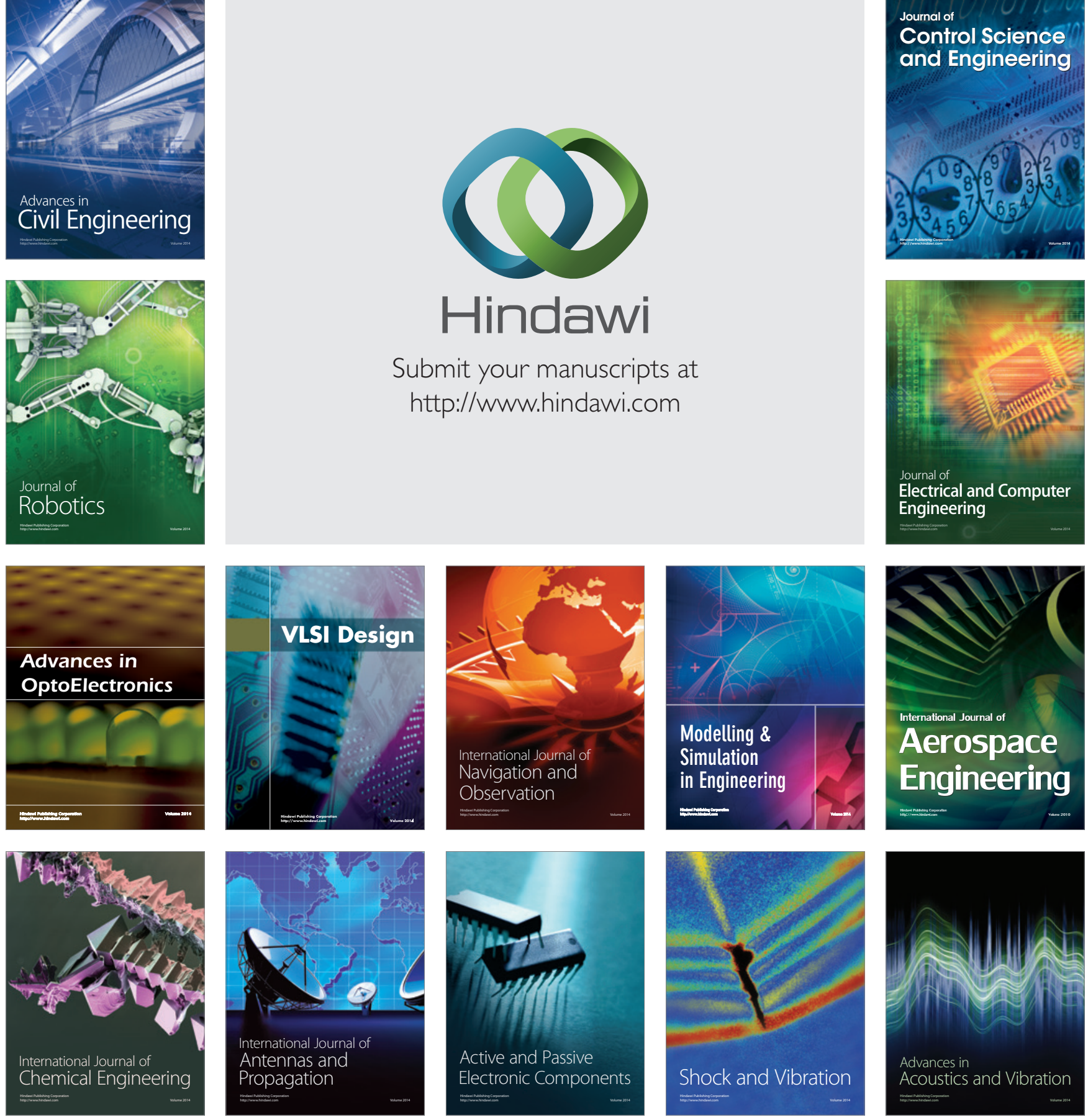